\title{
Threaded Connectors for Sandwich Pipes - Part 2: Optimisation of Stress Relief Groove
}

Ikechukwu Onyegiri, Maria Kashtalyan*

Centre for Micro- and Nanomechanics (CEMINACS), School of Engineering, University of Aberdeen, Fraser Noble Building, Aberdeen AB24 3UE

*Corresponding author: m.kashtalyan@abdn.ac.uk

\begin{abstract}
A concept for using snap-fit connectors in sandwich pipes is investigated numerically in two companion papers using a combination of $2 \mathrm{D}$ axisymmetric and 3D finite element models in Abaqus.

In the Part 1 paper, results of key parametric studies related to the installation analysis of sandwich pipes in deepwater are reported. The modification of the nib groove to include variable radii, the use of an elastomeric seal coupled with compressive pre-stress and an optimum resin-to-core ratio all proved to enhance the performance of the sandwich pipe snap-fit connectors. The influence of the interlayer adhesion configuration on the stress concentration experienced in the connector is also studied. Furthermore, a comparative study is performed to investigate the mechanical behaviour of the snap-fit connector concept in sandwich pipes and conventional pipe-in-pipe.

In the Part 2 paper, an optimisation study is carried out for the stress relief groove (SRG) in the pin of the snap-fit connector. A combined parameter is proposed to capture the relationship between the investigated geometric properties and the stress concentration factor at the SRG. It is established that the fillet radius could indeed be used to offset the drop in performance associated with increasing the SRG depth while improving the fatigue characteristics of the connector threads.
\end{abstract}

\section{Keywords}

Pipe joining, finite element modelling, snap-fit connector 


\section{Introduction}

Threaded and coupled (T\&C) connectors have been utilised in offshore oil pipelines with a good track record and serve economically well especially in shallow waters. They can be viewed as an alternative joining method for pipelines and risers in deepwater, where increased wall thickness and use of high strength steels could make welding a less favourable option.

It is well known that seal integrity and uniform stress distribution along the thread roots remain key challenges for threaded connectors especially when placed under severe bending loads (Sches et al. 2008). There exist a wide variety of "qualified" improvements to the conventional T\&C connector, mainly driven by enhanced mechanical performance for its use in risers, drill pipes and tendons. These improvements range from local to global geometrical modifications and a few are discussed in this paper. All improved designs seek to tackle a number of operational challenges depending on the joint requirements. The reduction of high local stresses and a more uniform stress distribution along the joint remain design priorities which when optimised lead to a better fatigue resistant connection.

This paper aims to identify the fatigue critical regions for a snap-fitted sandwich pipe and explore modifications to reduce the stress concentration in such regions. The relationship between the stress concentration at these regions and the connection's mechanical/geometrical properties is investigated for typical installation loads (bending, external pressure and axial loading). The formulation of optimization parameters to predict connection performance via parametric finite element analysis in Abaqus (Dassault Systèmes 2014) is also studied, in particular geometrical properties of the nib groove (considered in the Part 1 paper) and stress relief groove (considered in the present Part 2 paper). A better understanding of the added complexities to the snap-fit connector for its utilization in sandwich pipes is gained from the results from this study. As a complex multiaxial stress distribution is expected over the connection, the maximum principal stress is used.

\section{Trends in Connector Improvement}

A large variety of patented improvements to threaded and coupled pipe connectors follows two general trends, namely: modifications to the joint global geometry, (DeLange et al. 2003), (Verdillon 2004) and modifications to the thread profile, (Gunderson et al. 1990), (DeLange et al. 1999), (Flemming 2004),(Noel and Roussie 2004). 
Under installation loads, a conventional threaded connection will have peak stresses located at the root of the first engaged thread (FET) of the pin and last engaged thread (LET) of the box (Tafreshi 1999).

Local stiffness reduction to enhance uniform loading in the joint has been utilised in most solutions with numerical and experimental tests documented to verify. This approach is guided by easing the transfer of load between joint components at identified regions of local high stresses. (Bodine 1961) utilised this approach by applying a groove at the outer surface of the coupler box which is radially aligned with the LET of the pin. This would lead to a reduction in stiffness around that section, thus making it easier to deform elastically and transfer load more uniformly across the joint length. Longer engaged threads were proposed by both (DeLange et al. 2003) and (Verdillon 2004) with modifications to include thread run-out allowing for partial engagement of threads. This allows for some level of stress redistribution around the LET and as thus reduces the high local stresses that would normally have occurred.

It is well known that modifying the thread profile (especially at the root), could lead to an optimal design to reduce unwanted high stress regions in the connection. A conventional thread profile is defined by its height, flank angle, root groove finishing, pitch, load flank interference and fabrication tolerances, ASME B1.20.1(2013); leading to a pool of variables to combine in aim of arriving at an optimal design. This is no mean feat as some designs although showing an improvement in fatigue properties for the connector, require unattainable fabrication tolerances at that scale, (Van Wittenberghe et al. 2011). This creates a hurdle for this approach from an economic and technical point of view.

Nevertheless, significant improvements have been made to standard thread profiles. Take for instance, an early modification by (Saunders et al. 1985) which adopted a larger radii and stress relief groove at the root coupled with a modified pin profile pitch to improve load distribution via radial interference. (DeLange et al. 2003) took the approach of designing with a zero degree load flank and increased root radii with the goal of significantly reducing the radial component which contributes to pin-box thread separation. (Banker et al. 1994) patented an improved fatigue connector with load flanks having rounded corners while also designing the thread profile with a negative load flank which through research by (Takano et al. 2002) showed improved resistance to plastic yielding, galling and increased tensile, bending and external pressure capacity. Modifications by (Noel and Roussie 2004) took the approach of grooving the FET and LET of a buttress type thread in aim of reducing the stiffness and creating multiple stress zones which in turn improves load distribution around the FET and LET region. (Nakamura et al. 2011) modified their design by using 
two-step stabbing flanks for the threads in aim of improving pin insertion and galling resistance. (Sches et al. 2008) came up with a threaded profile, in which the gap between the loading and stabbing flanks was closed, increased the root radii, increased the chamfer angle on the stabbing flank and moved surface contact from the pin thread root. The resulting profile was reported to be able to isolate the effects of fretting from the effects of fatigue loading.

Another proven approach to improving connector performance is the inclusion of stress relief features in the joint geometry. According to API 7-1 (2006), stress relief features can be described as "a modification performed on rotary shouldered connections by removing the unengaged threads on the pin or box to make the joint more flexible and to reduce the likelihood of fatigue-cracking in highly stressed areas". The two most common features are: the stress relief groove (SRG) and the bore back (BB) as shown in Fig. 1. Stress relief features will cause a slight reduction in the tensile strength of the pin and the section modulus of the connection. However, under most conditions this reduction in cross-sectional area is more than offset by the improvement of the connector under fatigue loading, hence its acknowledged advantage (Bailey et al. 1994). In other words, this leads to a reduction in stiffness at the groove region and as such is elastically deformed preferably to lower the stress concentration that normally occurs in the LET (Tafreshi et al. 1993; Tafreshi 1999). revealed that the use of SRG and BB in a standard NC46 and NC50 connector indeed had advantages in reducing the stress concentration in T\&C connector under axial, bending and torsion loads.

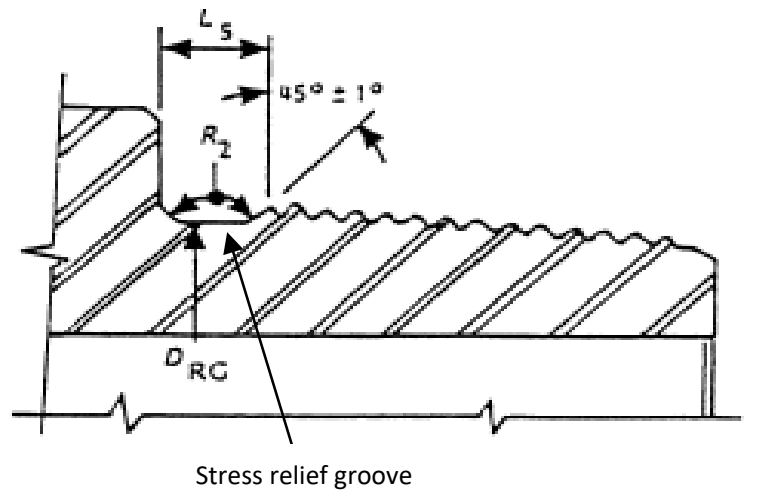

Figure 1 Geometry of a standard connector: (left) Pin with stress relief (right) Box with bore back (excerpt from (Tafreshi 1999))

No doubt the adaptation of stress relief grooves into the design of T\&C connectors has been welcomed and its effectiveness in prolonging connector life established (Pallini et al. 2002) (Pollack and Riggs 2011). (API-RP-7G 1998) draws conclusions via experimental fatigue testing under service conditions that an optimal stress reduction can be achieved in an NC50 connection with a relief groove width of approximately 1 -inch. (Hommel 2000) also agreed with this optimal width by carrying out a parametric study on a 5-1/2 F.H connector, also concluding that the engagement of a 
partial box thread rather than a full thread with the LET of the pin has a significant advantage by lowering the stress in that section of the pin.

It should be emphasized at this point that although T\&C connector design is properly standardised for drill pipes and to some extent for risers, when it comes to its application for oil and gas pipelines, proprietary joint types rule the market space. Some would argue that this is due to the uniqueness of multiphase oil and gas transport and flow conditions while some would blame it on a weightier industry confidence on welding as a preferred joining method for pipelines. This on the other hand has helped forge a new business line for joint designers who seek to develop new and modified systems to make economic profit. At the moment, threaded connectors for flowlines are certified by ISO 21329 (2004) and follow strict adherence to operational health and safety.

\section{Stress Relief Groove}

It is well known from standards (API-RP-7G 1998) and numerical analysis (Tafreshi et al. 1993) (Tafreshi 1999) (Hommel 2000) (Pallini et al. 2002) that the stress relief groove and bore back represent an effective modification to threaded line pipe joints leading to improved fatigue performance and prolonged connector life. The pathway to this solution involves reducing the maximum SCF in the connector which is generally known to be at the LET of the pin threads. The influence of this solution at the critical LET of the pin as can be seen in Fig. 6a of the Part 1 paper; this was also adopted in the snap-fit connector design for sandwich pipes. For elastic analysis, the truncation height for the connector threads had no significant effect on the SCF at the thread roots as the SCF was calculated as a function of the root diameter.

The stress relief groove design for an 8-inch threaded pipe is shown in Fig.2a. To achieve geometric convergence some dimensions were made consistent with (ASME B1.20.12013) standard dimensions or derived as function of such dimensions. The essential geometric parameters describing the stress relief groove to be used in this study are shown in Fig. $2 \mathrm{~b}$. 


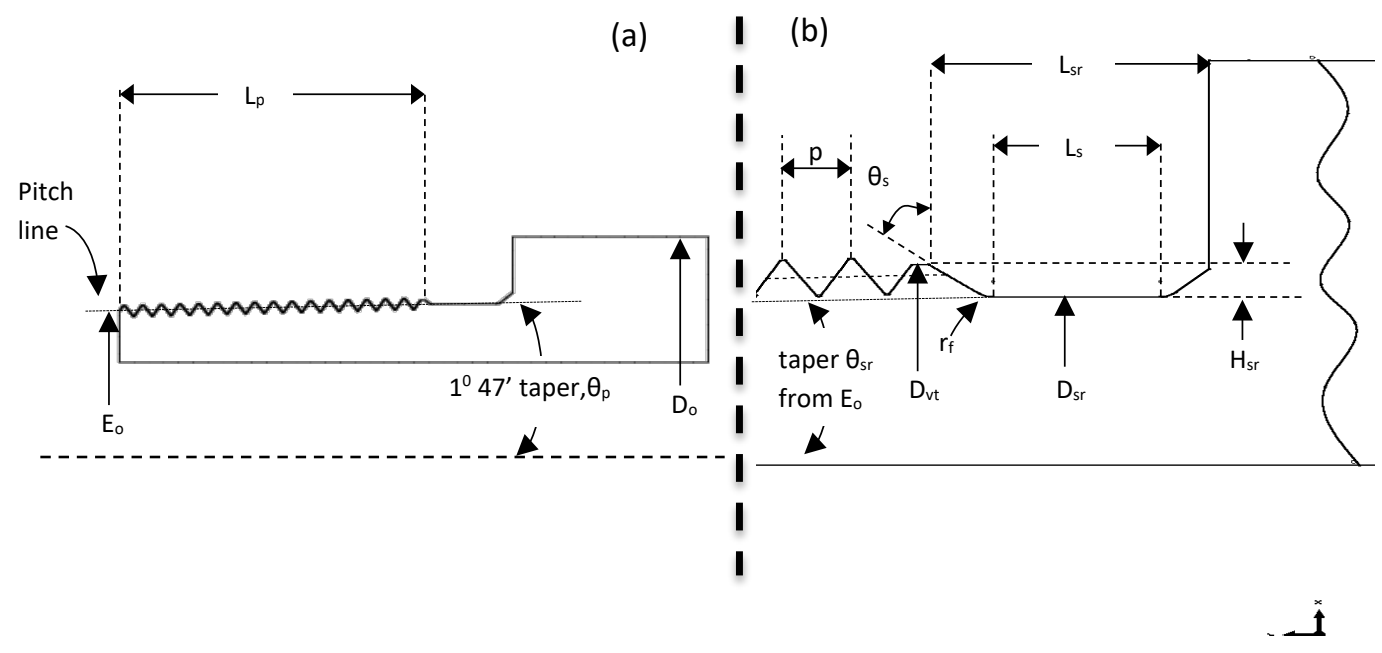

Figure 2 Essential geometry parameters for: (a) Line pipe threads (b) SRG

Parameter description and expression is shown in Table 1. As is seen, some parameters are made functions of others. An elastic material model was used with properties of API X65 according to (APIAPI-5L 2004). The material was modelled as elastic-perfectly plastic. The material's yield stress was set at $448 \mathrm{MPa}$, elastic modulus at $207 \mathrm{GPa}$ and Poisson ratio of 0.3 . As the study focused mainly on the LET and SRG, only six fully engaged threads were used (Fig.3a) and the axial load scaled down to $30 \mathrm{MPa}$. Makeup was modelled by using a simple tie constraint at the inner abutment face which proved sufficient as the load applied was not above the jump-out strength of the connector as estimated by (Clinedinst 1965). The pin end was fixed while the axial load was applied at the box end with contact properties defined about the pin and box threads using a coefficient of friction (COF) of 0.1. In elastic analysis, it has been proven that the COF doesn't significantly affect the stress and load distribution patterns within the connector (Hommel 2000) but being a property that defines surface forces, (Van Wittenberghe et al. 2011) showed that the COF has somewhat of an inverse relationship with thread opening, meaning that using an appropriate COF in modelling threaded connectors is necessary. Again the maximum principal stress (axial) is used as the measure for reported stress in this study which is complaint as studied by (Chang and Nijar 2008) and is in concordance with the direction of loading. 


\begin{tabular}{|l|l|l|}
\hline Parameter & Description & Expression \\
\hline$E_{o}$ & Pitch diameter at thread start & $214.21(\mathrm{~mm})$ \\
\hline$L_{p}$ & Overall thread length & $54.5(\mathrm{~mm})$ \\
\hline$D_{o}$ & Coupler outer diameter & $219.075(\mathrm{~mm})$ \\
\hline$\theta_{p}$ & Pitch line taper angle from $E_{0}$ & $1.8(\mathrm{deg})$ \\
\hline$p$ & Pitch & $3.173(\mathrm{~mm})$ \\
\hline$\theta_{s r}$ & SRG depth taper angle from $E_{0}$ & Varied (deg) \\
\hline$H$ & Basic thread height & $1.88(\mathrm{~mm})$ \\
\hline$r_{f}$ & SRG fillet radius & Varied $(\mathrm{mm})$ \\
\hline$L_{s r}$ & SRG length & Varied as function of $p(\mathrm{~mm})$ \\
\hline$\theta_{s}$ & SGR flank angle & Varied $(\operatorname{deg})$ \\
\hline$D_{s r}$ & SRG outer diameter & $E_{o}+2 L_{p} \tan \theta_{s r}(\mathrm{~mm})$ \\
\hline$D_{v t}$ & Vanish thread diameter & $E_{o}+2 L_{p} \tan \theta_{p}+0.8 H(m m)$ \\
\hline$H_{s r}$ & Depth of SRG & $\left(D_{v t}-D_{s r}\right) 0.5(m m)$ \\
\hline$L_{s}$ & SRG truncated width & $L_{s r}-2 H_{s} \tan \theta_{s}-0.42 r_{f}(m m)$ \\
\hline
\end{tabular}

\section{Stress Relief Groove Optimisation}

\subsection{Scripting of Parametric Studies}

Although the thread profile of the snap-fit connector is kept constant through the study, in order to carry out the parametric study, numerous FE runs have to be conducted due to the changing geometry of the SRG with varying parameters. In the absence of large computational resources, submodelling would have to be used to automatically regenerate the joint geometry for every change in geometric parameters although this method can be cumbersome and time consuming especially for complex geometries. In this study, a high performance cluster was used which had more than required computational power to allow us to run the simulations faster. As such, the parametric models were automatically generated, inputted and run by the use of Python scripting and Abaqus PDE which was linked to the cluster using a SLURM script (Yoo et al. 2003).

The automatic input was executed from the sketch module and input files recorded in a batch file for execution. All extension plugins used in this study were compatible with Abaqus 6.14 and as such custom plugins were not needed. Sketch constraints ensured that a closed profile was generated for every design case considered. The models were meshed with linear axisymmetric elements instead 
of quadratic elements due to the existence of contact non-linearity between the threads for this study (Chang and Nijar 2008). For contact non-linearity in Abaqus, the greater the number of adjusted nodes and their relative displacements, the more likely the convergence issues. Although second order elements provide higher accuracy, for contact conditions with severe element distortion, the mid-nodes can generate abnormal stress patterns within the element integration points. The trade-off being the generation of large number of elements to avoid convergence issues. Mesh constraints were applied as single and double bias (Abaqus terminology) across connector assembly and geometric partitions ensured an evenly distributed mesh profile as seen for the last engaged thread and stress relief groove fillet in Figure $3 \mathrm{~b}$ with 24 elements assigned to both the SRG fillet and the root of the LET. Mesh sensitivity studies revealed that above 24 elements there was no significant change in the instability peak stresses experienced at the two regions derived by analysing using the RIKS method. Due to the partition pattern required for mesh convergence of the automatically generated parametric models, the number of elements at both regions needed to be the same. Mesh size was automatically constrained by approximate global size with custom seeds (which are regenerated depending on the parameter varied) assigned to the SRG region.
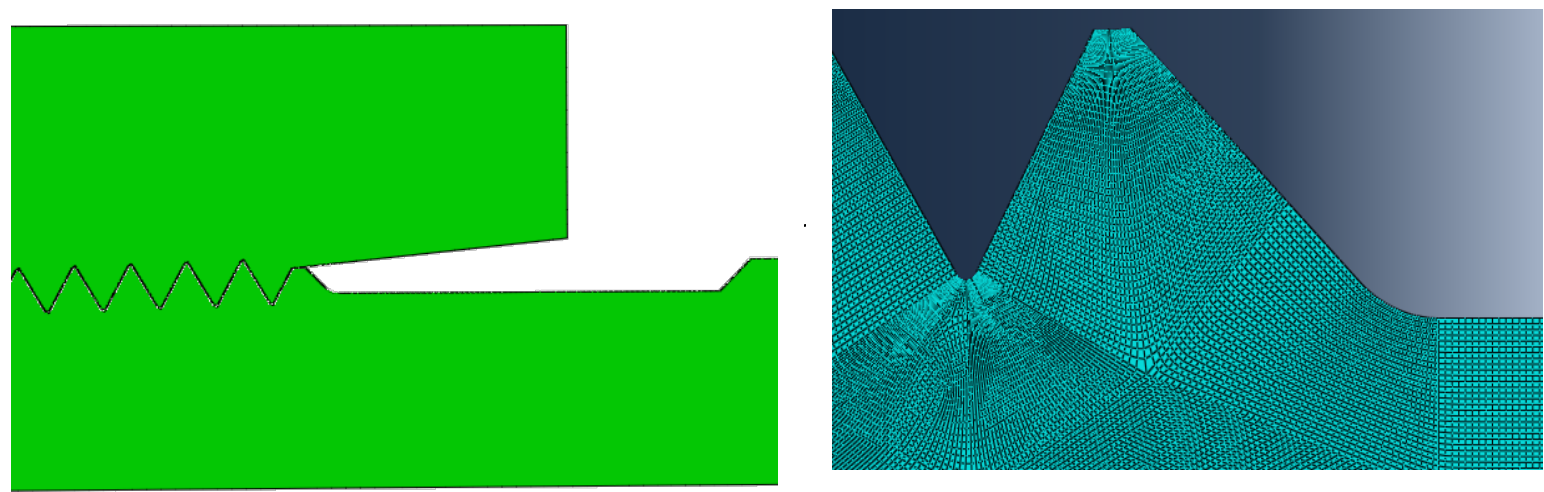

Figure 3 (left) FEA geometry (right) Mesh profile for LET and SRG

Mesh sensitivity studies were carried out to ascertain the value for the maximum deviation factor at which the peak stresses became less significantly dependent on the curvature control. As the fillet region of the SRG was identified as the location for the peak stresses in the groove, mesh constraints were also applied to maintain elements with aspect ratios that do not lead to the creation of distorted elements and analysis errors.

The influence of varying geometric parameters on the peak stresses at the LET and SRG was studied by the use of Abaqus scripting via Python programming language. Parameters as defined in Table 1 were used to develop design sets as defined in Table 2. Firstly, models observed the influence of changing just one parameter and then the influence of changing multiple parameters simultaneously. For all design sets, the automated scripts could not accommodate geometrical 
functions created by varying more than three parameters. Multiple constraints had to be adopted in the geometry sketch files to restrict dimensions within the set functions and resolve geometrical convergence problems.

Table 2 Design sets and defining parameters

\begin{tabular}{|l|l|l|l|l|l|}
\hline Design set & 1 & 2 & 3 & 4 & 5 \\
\hline Varied & $\mathrm{L}_{s r}$ & $\theta_{s r}$ & $\theta_{s}$ & $\mathrm{r}_{f}$ & $\theta_{s r}, \mathrm{r}_{f}$ \\
\hline Fixed & $\theta_{s r}, r_{f}, \theta_{s}$ & $\mathrm{~L}_{s r}, r_{f}, \theta_{s}$ & $L_{s r}, r_{f}, \theta_{s r}$ & $L_{s r}, \theta_{s}, \theta_{s r}$ & $L_{s r}, \theta_{s}$ \\
\hline
\end{tabular}

From a geometric perspective, the depth and length of the SRG are arguably the most critical parameters when it comes to the magnitude of peak stresses. Increasing the depth of the SRG will reduce its wall thickness which will in turn increase the magnitude of peak stress. From study carried out by (Hommel 2000), it was found that indeed an optimal length exists for the SRG; lengths shorter than optimal introduced further stress concentrations while lengths longer than optimal have a tendency to load up the LET excessively. In order to reduce the number of studied parameters for geometric convergence, some variables were made a function of others as seen in Table 1 . The maximum stresses in the SRG always occurred at the fillet and there existed in most models an inverse relationship between the peak stresses at the fillet of the SRG and the peak stresses at the root of the LET of the pin. Two pipe sizes (6-inch and 8-inch) were considered in this study. The models adopted to investigate the effect of make-up (8-inch only) for this study were modified to have a larger pin outer diameter after the SRG termination. This was done to create a pin shoulder for the simulation of makeup. From the analysis results presented below, we can see the influence of the shoulder on the SCF. For example, in Fig.4, we notice that the SCF of the no makeup case in (b) is lower than that of the 8-inch model in (a).

\subsection{Influence of Groove Depth}

The influence of the groove depth, $\mathrm{H}_{\mathrm{sr}}$ on the peak stresses was studied by varying the SRG depth taper angle (from $E_{\mathrm{o}}$ ) $\theta_{\mathrm{sr}}$. As expected, an increase in the depth of the groove increases the magnitude of peak stress in the SRG for both the 6-inch and 8-inch models as seen in Fig.4a. The length, fillet radius and flank angle of the SRG are kept constant for this design set. $\theta_{\mathrm{sr}}$ was varied from $2.0^{\circ}$ to $-0.9^{\circ}$ from $E_{o}$ and the corresponding groove depth computed by the expression in Table 1. From analysis result, keeping the SRG depth to a minimum would be a preferred solution to 
avoiding high peak stresses at the SRG but in turn would mean the peak stresses experienced at the LET would be higher as seen in Fig.4a, thus designing the SRG up to a depth as to avoid plastic deformation at the fillet would be preferred. For a case without makeup (Fig. 4b), the peak stress at the LET begins to increase at a depth at which the groove diameter is less than $E_{o}\left(\theta_{\text {sr }}\right.$ carries a negative value). This represents a groove length at which the stress amplification at the SRG fillet is large enough to cause higher stresses at the LET. This would clearly be a function of the distance between the LET and the SRG fillet.
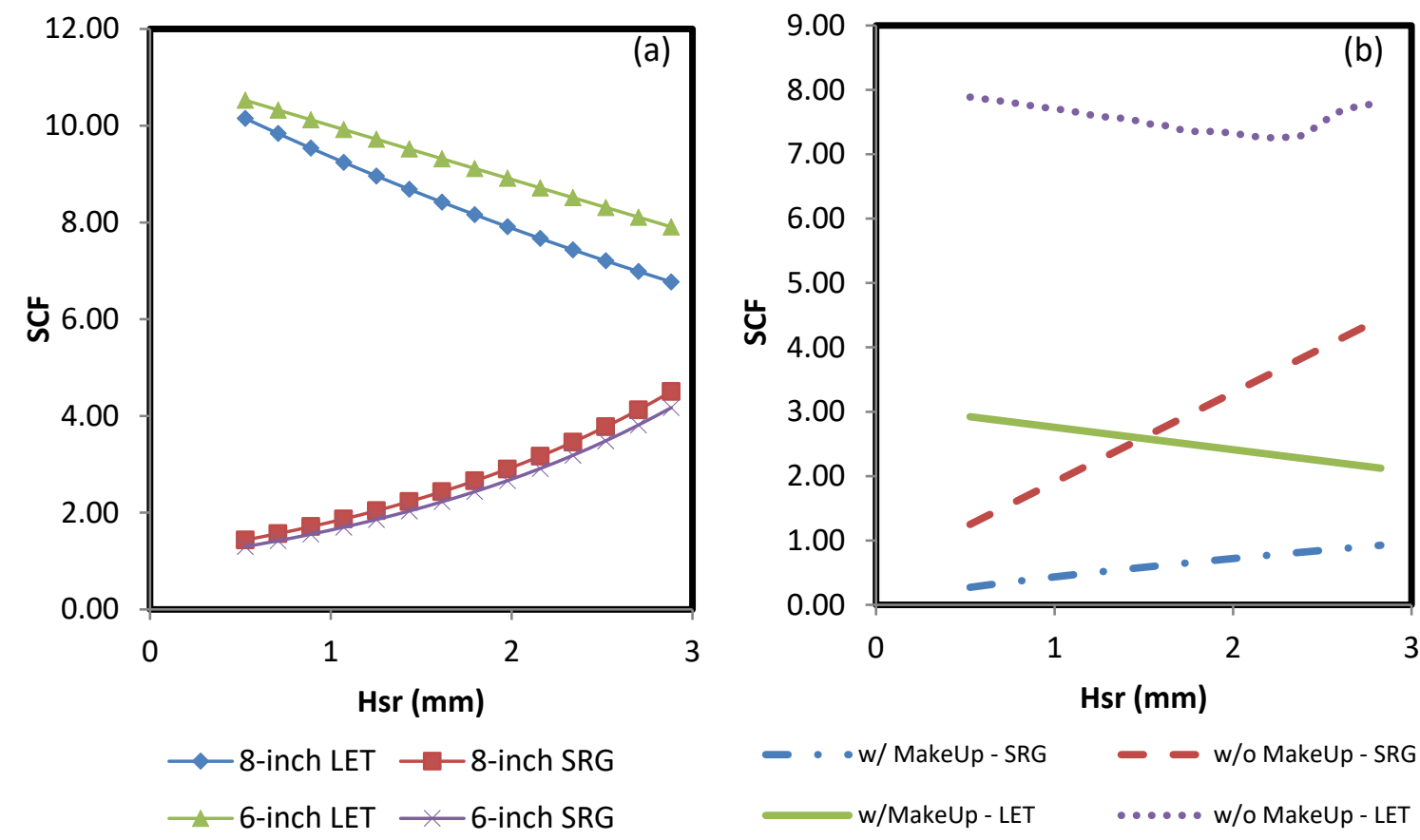

Figure 4 Influence of SRG depth on SCF (a) 8in,6in (b) Makeup, No makeup

\subsection{Influence of Groove Length}

The influence of the SRG length (design set 1 ) can be seen in Fig. 5 . The groove depth and fillet radius were kept constant. The length was varied from 10 times the pitch to the length of the pitch of the connector. As can be seen from the analysis of results, the peak stresses tend to be minimal at a certain value of depth to length ratio corresponding to about 1.5 times the pitch to 2.5 times the pitch. It goes to show that the length has a significant effect on the magnitude of peak stresses experienced at the SRG. In the absence of make-up, the peak stress at the SRG tends to increase as the length gets shorter (Fig.5a). This is due to the reduced load bearing area in the SRG. For a longer length than optimum, the pipe threads tend to experience additional loads brought about by the radial compressive stresses generated in the pin. 

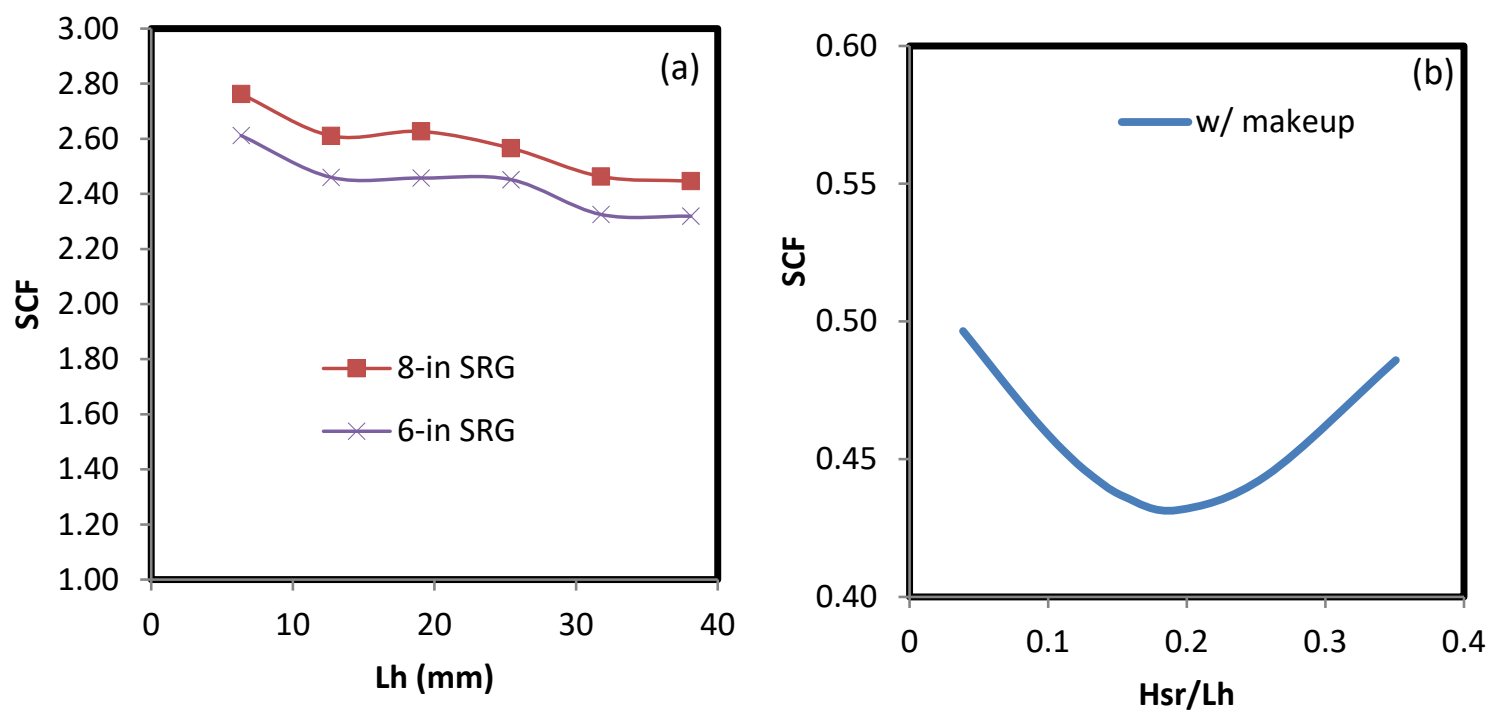

Figure 5 (a) Influence of SRG width for 8in, 6in (b) Influence of Hsr/Lh on SCF for makeup model

\subsection{Influence of flank angle and fillet radius}

The influence of the SRG flank angle $\theta_{s}$ (design set 3 ) on the peak stresses experienced at the SRG for a case with and without make-up can be seen in Fig. $6 \mathrm{a}$. The $\theta_{\mathrm{s}}$ was varied from $30^{\circ}$ to $60^{\circ}$ with all other parameters fixed. The variable fillet was made up of four $\operatorname{arcs}\left(r_{0}, r_{1}, r_{2}, r_{3}\right)$ with each arc covering a length equivalent to $\theta_{s} / 4\left(\theta_{s}\right.$ was set at $\left.45^{\circ}\right)$ where:

$$
\mathrm{r}_{0}=3 \mathrm{~mm}, \mathrm{r}_{1}=\mathrm{r}_{0}-\mathrm{d}, \mathrm{r}_{2}=\mathrm{r}_{0}+\mathrm{d}, \mathrm{r}_{3}=\mathrm{r}_{0}-\frac{\mathrm{d}}{2} \quad \mathrm{~d}=0.4 \mathrm{~mm}
$$

Increasing the fillet radius $r_{f}$ was seen to reduce the peak stresses at the SRG for both a case of make-up and no make-up as illustrated in Fig. 6b. As expected, one solution to reducing the stress concentration would be utilising a variable radius fillet and this is clearly shown in the results although variable radius fillets tend to suffer from fabrication challenges and may require custom procedures making them unfavourable especially for small radius fillets. 

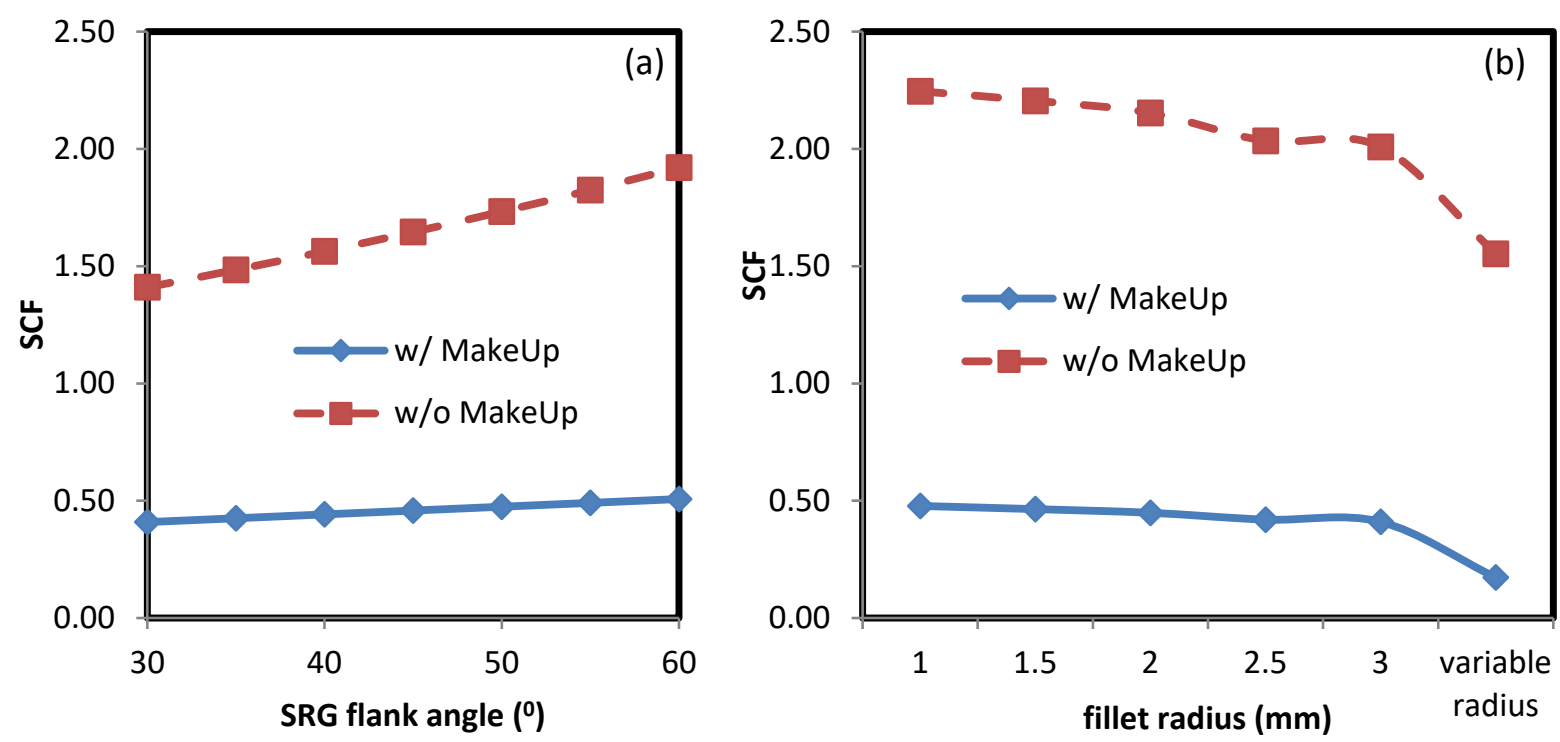

Figure 6 Influence of SRG (a) flank angle (b) fillet radius on SCF

\subsection{Parameter Combination Study}

The combined effect of varying the groove height, fillet radius and truncated length was studied to establish their influence on the peak stresses experienced (design set 5). Parameter $r_{f}$ was varied from $0.5 \mathrm{~mm}$ to $8 \mathrm{~mm}, \theta_{\mathrm{sr}}$ varied from $1.8^{\circ}$ to $-0.78^{\circ}$ relative to $E_{0}$ and $L s$ computed from its given function in Table 1. The parameter ranges were constrained so as to ensure convergence of the model sketches. The influence was studied using a unique parameter $\alpha$ described by the expression:

$$
\alpha=\frac{\mathrm{H}_{\mathrm{sr}}^{\beta}}{\mathrm{L}_{\mathrm{s}}}
$$

where $\beta$ is a normalising parameter defined by the expression:

$$
\beta=\frac{D_{s r}^{2}}{D_{s r(i)}^{2}}
$$

$D_{S r}$ is the outer diameter of the SRG computed from:

$$
\mathrm{E}_{0}+2 \mathrm{~L}_{\mathrm{p}} \tan \theta_{\mathrm{sr}}(\mathrm{mm})
$$

$D_{\text {sr(i) }}$ is the outer diameter of the SRG when $\theta_{\text {sr }}=\theta_{p}$

From the analysis results shown in Fig. 7a, we can see that the depth of the SRG weighs more than the fillet radius for the range of parameters selected. Although as $r_{f}$ increases we notice its improved significance on the peak stresses at the SRG. This is more significant at a value of $r_{f}=6 \mathrm{~mm}$ (broken line) where the fillet radius is large enough to counter the influence of the increased groove depth. This is defined by a region at which $\alpha \geq 0.35$ and design points at which $r_{f} \geq 0.875 \mathrm{~L}_{\mathrm{s}}$. It can be seen that the peak stresses become fairly constant even as the depth increases. 
Fig. $7 \mathrm{~b}$ shows the influence of the combined parameters on the stress concentration factor at the LET. As expected with an increase in peak stresses at the SRG, the SCF at the LET reduces slowly and steadily.
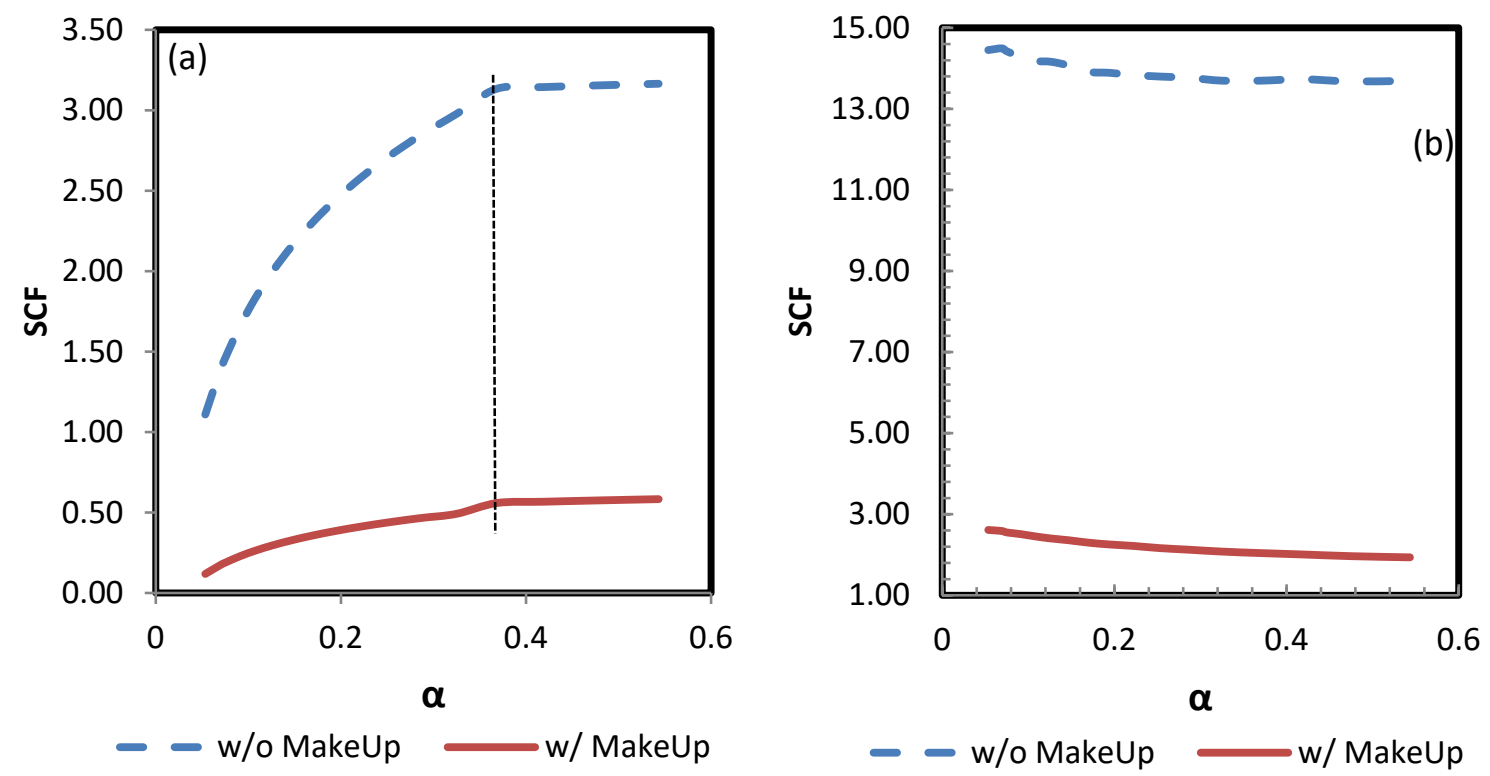

Figure 7 Influence of combined parameters on SCF for makeup and no makeup cases (a) SRG (b) LET

\section{Conclusions and Further Work}

The investigation into performance enhancing geometric and mechanical modifications to snap-fit connectors for sandwich pipes has been carried out. Firstly, regions susceptible to high stress concentrations under a combination of loadings were identified. Thereafter, with the aid of FE modelling, we were able to highlight some trends that would lead to an improved performance for the snap-fit connector, see conclusions of the Part 1 paper.

In the Part 2 paper, an optimisation study was carried out for the stress relief groove adopted in the pin of the snap-fit connector. This was made possible by 2D axisymmetric model with Python scripting via Abaqus. For most of the models, an increase in the peak stresses at the SRG directly resulted in a reduction in the peak stresses at the critical LET. This study considered only axial loading and analysis results revealed that increasing the SRG depth would lead to an increase in the peak stresses at the SRG. The results also showed that the size of the pipe didn't have any significant effect on the load and stress distribution in the connector for axial loading carried out using elastic FE analysis. It was observed that an SRG optimal length exists but only for a case where makeup is present. Increasing the SRG fillet radius and flank angle would reduce the SCF at the SRG although utilising a variable radius fillet would be most beneficial. A combined parameter was created to 
capture the relationship between the investigated geometric properties and the SCF at the SRG and it was discovered that the fillet radius could indeed be used to offset the drop in performance associated with increasing the SRG depth. The design point at which this happens was found to be at $r_{f} \geq 0.875 L_{s}$ and a combined parameter value greater than or equal to 0.35 . This analysis was carried out using an 8 in API threaded line pipe and further work will have to be carried out to validate the study for other line sizes. Future work is planned to carry out this study under pure bending and combined loading to get a better understanding the influence of the SRG geometry on the joint performance.

\section{Acknowledgements}

The authors would like to acknowledge the financial support of the University of Aberdeen, through the Elphinstone PhD studentship, and the support of the Maxwell computer cluster funded by the University of Aberdeen. 


\section{References}

API-5L (2004) Specification of Line Pipe, API Publishing Services: American Petroleum Institute.

API-RP-7G (1998) Recommended Practice for Drill Stem Design and Operating Limits, 16th Edition, API Publishing Services: American Petroleum Institute.

ASME-B1.20.1 (2013) Pipe Threads, General Purpose, Inch: American Society of Mechanical Engineers.

Bailey, J.R., McBride, R.P., Moyer, M.C. and Day, J.B., Exxon Production Research Company (1994) Drill collar connections, USH1329 H.

Banker, E.O., Suzuki, T., Klementich, E.F. and Bouche, J.K., Nkk Corporation, Marubeni Tubulars, Inc. (1994) Buttress-threaded tubular connection, US5358289 A.

Bodine, A.G. (1961) Sonic well pump tubing string, US2992613.

Chang, R. and Nijar, A. (2008) 'Threaded and Coupled Connector Analysis Using Abaqus CAXA', in Abaqus Users' Conference, Newport, Rhode Island, May 19-22, Dassault Systèmes 2008.

Clinedinst, W.O. (1965) 'Strength of Threaded Joints for Steel Pipe', Journal of Engineering for Industry, 87(2), 125-134.

DeLange, R.W., Evans, E. and Buster, J.L., Grant Prideco Inc. (1999) Threaded connection for enhanced fatigue resistance, US 5931511.

DeLange, R.W., Richard, W. and Evans, E.M., Grant Prideco L. P. (The Woodlands, TX) (2003) Threaded and coupled connection for improved fatigue resistance, 6609735.

Flemming, F. (2004) String of drill pipes for rotary percussion drilling, especially for simultaneous drilling, EP1117897.

Gunderson, R.H., Burns, J.Q. and Fox, S.A., Google Patents US 4892337 (1990) Fatigue-resistant threaded connector, US 4892337.

Hommel, M. (2000) 'Optimization of Stress-Relief Grooves for Rotary Shoulder Connections', in IADC/SPE Drilling Conference, New Orleans, Louisiana, 2000/1/1/, SPE: Society of Petroleum Engineers SPE-59141-MS.

Nakamura, K., Hamamoto, T., Sugino, M. and Yamaguchi, S., Sumitomo Metal Industries, Ltd. (Osaka, JP) (2011) Threaded joint for steel pipes, 7900975. 
Noel, T. and Roussie, G., Google Patents (2004) Fatigue-resistant threaded component for a tubular threaded joint, US20040155465.

Pallini, J.P., Lyle, R.D. and Munk, B.N., Abb Vetco Gray Inc (2002) Threaded connector, US6478344 B2.

Pollack, J. and Riggs, D.C. (2011) 'Improved Concentric Thread Connectors for SCRs and Pipelines', in Offshore Technology Conference, Houston, Texas, USA, 2011/1/1/, OTC: Offshore Technology Conference.

Saunders, D.D., Kalsi, M.S. and Chen, G.S., Google Patents (1985) Tool joint, US4549754 A.

Sches, C., Massaglia, J. and Desdoit, E. (2008) 'Fatigue resistant threaded and coupled connectors for deepwater riser systems: Design and performance evaluation by analysis and full scale tests', 5, 407-420.

Tafreshi, A. (1999) 'SIF evaluation and stress analysis of drillstring threaded joints', International Journal of Pressure Vessels and Piping, 76(2), 91-103.

Tafreshi, A., Tafreshi, W.D. and Dover, W.D. (1993) 'Stress analysis of drillstring threaded connections using the finite element method', International Journal of Fatigue, 15(5), 429438.

Takano, J., Yamaguchi, M. and Kunishige, H. (2002) Development of premium connection KSBEAR for withstanding high compression, high external pressure, and severe bending: JFE Steel Corporation.

Van Wittenberghe, J., De Baets, P., De Waele, W., Galle, T., Bui, T.T. and De Roeck, G. (2011) 'Design characteristics that improve the fatigue life of threaded pipe connections'.

Verdillon, L., Vallourec Mannesmann Oil \&amp; Gas France (Aulnoye-Aymeries, FR), Sumitomo Metal Industries. Ltd. (Osaka, JP) (2004) Threaded tubular element for fatigue resistant threaded tubular joint and resulting threaded tubular joint, 6729658.

Yoo, A.B., Jette, M.A. and Grondona, M. (2003) 'SLURM: Simple Linux Utility for Resource Management', in Feitelson, D., Rudolph, L. and Schwiegelshohn, U., eds., Job Scheduling Strategies for Parallel Processing, Berlin, Heidelberg, 2003, Springer Berlin Heidelberg, 44-60. 